\title{
BMJ Open Observational study of the safety of buprenorphine+naloxone in pregnancy in a rural and remote population
}

\author{
Naana Afua Jumah, ${ }^{1}$ Craig Edwards, ${ }^{2}$ Jazmyn Balfour-Boehm, ${ }^{1}$ \\ Kassandra Loewen, ${ }^{3}$ Joseph Dooley, ${ }^{4}$ Lianne Gerber Finn, ${ }^{4}$ Len Kelly ${ }^{3}$
}

To cite: Jumah NA,

Edwards C, Balfour-Boehm J, et al. Observational study of the safety of buprenorphine +naloxone in pregnancy in a rural and remote population. BMJ Open 2016;6:e011774. doi:10.1136/bmjopen-2016011774

- Prepublication history for this paper is available online. To view these files please visit the journal online (http://dx.doi.org/10.1136/ bmjopen-2016-011774)

Received 9 March 2016 Revised 4 August 2016 Accepted 1 September 2016

CrossMark

For numbered affiliations see end of article.

Correspondence to Dr Naana Afua Jumah; njumah@nosm.ca

\section{ABSTRACT}

Objectives: To describe the effect of in utero exposure to the buprenorphine+naloxone combination product in a rural and remote population.

Setting: A district hospital that services rural and remote, fly-in communities in Northwestern Ontario, Canada.

Participants: A retrospective cohort study was conducted of 855 mother infant dyads between 1 July 2013 and 30 June 2015. Cases included all women who had exposure to buprenorphine+naloxone during pregnancy $(n=62)$. 2 control groups were identified; the first included women with no opioid exposure in pregnancy $(n=618)$ and the second included women with opioid exposure other than buprenorphine tnaloxone $(n=159)$. Women were excluded if they had multiple pregnancy or if they were part of a methadone programme $(n=16)$. The majority of women came from Indigenous communities.

Outcomes: The primary outcomes were birth weight, preterm delivery, congenital anomalies and stillbirth. Secondary neonatal outcomes included gestational age at delivery, Apgar scores at 1 and $5 \mathrm{~min}$, NAS Score $>7$ and treatment for neonatal abstinence syndrome (NAS). Secondary maternal outcomes included the number of caesarean sections, postpartum haemorrhages, out of hospital deliveries and transfer of care to tertiary centres.

Results: No difference was found in the primary outcomes or in the Apgar score and caesarean section rate between in utero buprenorphine+naloxone exposure versus no opioid exposure in pregnancy. Compared to women taking other opioids, women taking buprenorphine+naloxone had higher birthweight babies $(p=0.001)$ and less exposure to marijuana $(p<0.001)$ during pregnancy.

Conclusions: Retrospective data suggest that there likely is no harm from taking buprenorphine+naloxone opioid agonist treatment in pregnancy. Larger, prospective studies are needed to further assess safety.

\section{INTRODUCTION}

Opioid dependence in pregnancy is an increasingly common occurrence in rural and remote areas such as Northwestern

\section{Strengths and limitations of this study}

- Opioid misuse is epidemic in rural and remote areas of Northwestern Ontario, Canada, with up to $30 \%$ of women exposed during pregnancy. Community-based buprenorphine+naloxone programmes have engaged many rural women in treatment programmes who otherwise would not receive care.

- This is the largest cohort of women exposed to buprenorphine+naloxone in pregnancy and contains detailed information about the daily dose, cumulative dose and exposure time with respect to each trimester of pregnancy.

- While 62 women had exposure to buprenorphine +naloxone in pregnancy, only 3 women had exposure throughout all three trimesters, a further $48 \mathrm{had}$ exposure in the first trimester only and the remainder had variable lengths of exposure.

- Data on illicit substances, smoking and alcohol use during pregnancy were determined by selfreport and confirmed with urine drug screens. Data were not collected on other exposures or on the use of other medications such as antidepressants, anxiolytics and folic acid.

- Data were collected retrospectively and were limited to antenatal, maternal and neonatal outcomes. Prospective data and long-term outcomes would provide more robust safety data.

Ontario, Canada, where up to $28 \%$ of pregnancies are exposed to opioid use. ${ }^{1}$ Our catchment area of 30000 patients includes 25000 patients in remote communities who are mostly Indigenous and receive their initial pregnancy care at the nursing station in their community. ${ }^{2}$ Methadone treatment has the most evidence regarding safety and efficacy in pregnancy; ${ }^{3}{ }^{4}$ however, due to logistical and regulatory limitations, methadone is often not available in rural and remote areas. ${ }^{5}$ Community-based, sublingual buprenorphine+naloxone treatment programmes have been established in rural and remote, predominantly Indigenous 
communities in order to provide access to treatment in areas with high rates of opioid dependence and no access to methadone. ${ }^{5}$

The WHO and several national Obstetrics and Gynaecology associations recommend that, when pregnancy is diagnosed, women participating in a buprenorphine+naloxone treatment programme switch to the buprenorphine mono-product because the safety of buprenorphine+naloxone has not been demonstrated in pregnancy. ${ }^{6-8}$ A multicentred randomised controlled trial demonstrated that the buprenorphine monoproduct has similar pregnancy outcomes and decreased severity of neonatal abstinence syndrome compared to methadone. ${ }^{9}$ Naloxone was added to buprenorphine as a deterrent to illicit use as it precipitates withdrawal from opioids when administered intravenously or intranasally but not via the buccal or sublingual routes. ${ }^{10} 11$ Precipitated withdrawal has been shown to result in adverse pregnancy outcomes. ${ }^{12} 13$

The caution against using buprenorphine+naloxone in pregnancy is not limited to concerns for withdrawal but also possible teratogenicity. However, to date, there have been no reports of teratogenicity in humans or animals. ${ }^{14}$ Congenital anomalies are only one marker of drug safety in pregnancy. The live birth rate, spontaneous abortion rate and stillbirth rates are also markers of safety, and among live births, preterm delivery, low birth weight and functional deficits are factors that may be affected by a medication. ${ }^{15}$ Further, the severity of pregnancy outcomes may be modified by the duration and intensity of the exposure to the medication.

In Canada, the buprenorphine mono-product is available only through a special access programme. In our setting, women are counselled to transition from buprenorphine+naloxone to either buprenorphine or longacting morphine when they present for antenatal care. As a result of delays in obtaining buprenorphine through the special access programme, many women are exposed to buprenorphine+naloxone during early pregnancy and into the second trimester. In addition, for personal reasons, some women opt to remain on buprenorphine+naloxone throughout their pregnancy. This study documents the pregnancy outcomes of a cohort of women from rural and remote communities in Northwestern Ontario who continued to take buprenorphine+naloxone treatment during pregnancy as part of a community-based treatment programme.

\section{METHODS}

\section{Participants}

Maternal and neonatal data were collected from outpatient antenatal clinic records and inpatient medical records for all pregnancies between 1 July 2010 and 31 July 2015. Cases included all women who had exposure to buprenorphine+naloxone during pregnancy. Two control groups were identified; the first included women with no opioid exposure in pregnancy and the second included women with opioid exposure other than buprenorphine+naloxone. All women who were receiving opioid agonist treatment with buprenorphine+naloxone were advised to switch to the buprenorphine mono-product when available, once pregnancy was diagnosed as per national guidelines. ${ }^{7}$ Cases represent those women who elected to stay on buprenorphine+naloxone during their pregnancy. Women were excluded if they had a multiple pregnancy or were taking methadone as part of a treatment programme. All infants room-in with their mother following delivery unless there is a medical or safety reason that precludes rooming-in. The majority of women came from Indigenous communities.

\section{Data collection}

A standard case report form was used to collect maternal and neonatal data. The maternal case report form contained information on the health and pregnancy history; smoking, drug and alcohol exposure; and intrapartum data. Smoking was defined by self-reported daily use of cigarettes and was further characterised by the number of cigarettes smoked per day. Alcohol and drug exposure were determined by self-report. Drug exposure was characterised further by urine drug screen results. The neonatal case report form contained information on birth weight, gestational age, Apgars, congenital anomalies and stillbirths.

The primary outcomes for the study was an assessment of the safety of buprenorphine+naloxone, including birth weight, preterm delivery (delivery prior to 37 +0 weeks gestational age), congenital anomalies and stillbirth. Secondary neonatal outcomes included gestational age at delivery, Apgar scores at 1 and 5 min, NAS Score $>7$ and treatment for NAS (two or more NAS Scores that are $>7$ ). NAS Scores were calculated using a modified Finnegan Scale at the bedside by nurses who have been trained to use this measure. Secondary maternal outcomes included the number of caesarean sections, postpartum haemorrhages, out of hospital deliveries and transfer of care to tertiary centres.

\section{Statistical analysis}

Categorical variables are presented as percentages while continuous variables are presented as a mean with SD. We compared cases to controls using a t-test for continuous variables and a Pearson $\chi^{2}$ test of independence or a Fisher's Exact test, as appropriate, for categorical data. ORs are presented with the $95 \%$ CI. Data analysis was performed with SPSS statistical software V.20 (SPSS, Chicago, Illinois, USA) and Microsoft Excel V.14.1.0 (Microsoft Corp, Redmond, Washington, USA). A p value $<0.05$ indicated statistical significance.

\section{Ethics}

Ethics approval was granted by the Sioux Lookout Meno Ya Win Health Centre Research Review and Ethics Committee. 


\section{RESULTS}

A total of 855 consecutive singleton births were included in the study, of these, 62 had exposure to buprenorphine+naloxone, 618 had no opioid exposure and 159 used illicit opioids during the pregnancy (figure 1). Sixteen women were excluded due to participation in a methadone treatment programme. Twenty-five women were excluded due to a multiple pregnancy. Maternal characteristics are described in table 1 . The overall rate of opioid exposure in pregnancy is $27.8 \%$. Data on the racial and ethnic make-up of our study participants as well as data on educational attainment were not collected. Previous studies of this population show that the majority $(85 \%)$ of women are Indigenous. ${ }^{2}$ Educational attainment among Indigenous women living on reserve is low where $57 \%$ do not complete high school, $16 \%$ receive a high school diploma, $19 \%$ participate in postsecondary education and only $4 \%$ have a university degree. $^{16}$

A total of 62 women had exposure to buprenorphine + naloxone in pregnancy (see figure 1). Of these women, three women continued prepregnancy buprenorphine +naloxone throughout pregnancy and after delivery. A further 48 women who were taking buprenorphine +naloxone prior to pregnancy switched to buprenorphine alone after the first trimester as per national and international guidelines. ${ }^{6-8}$ Eleven women were induced onto buprenorphine+naloxone during pregnancy, and this occurred in the first trimester for 6 women. The average daily dose of buprenorphine+naloxone was 8.2 $\pm 5.8 \mathrm{mg}$. Ongoing illicit opioid use was identified in 12 women $(19.4 \%)$ through routine urine drug screening. Six cases had positive urine drug screens for marijuana. No other illicit substances were identified on urine drug screening. Data were not collected for other psychotropic medications or antidepressant medications.

Cases and controls were of similar age at the time of delivery. However, women who had no opioid exposure in pregnancy had fewer pregnancies and fewer births compared to cases. The OR of Hepatitis C infection for women taking buprenorphine+naloxone compared to no opioid use was OR 15.7, 95\% CI 2.6 to 95.6. Women

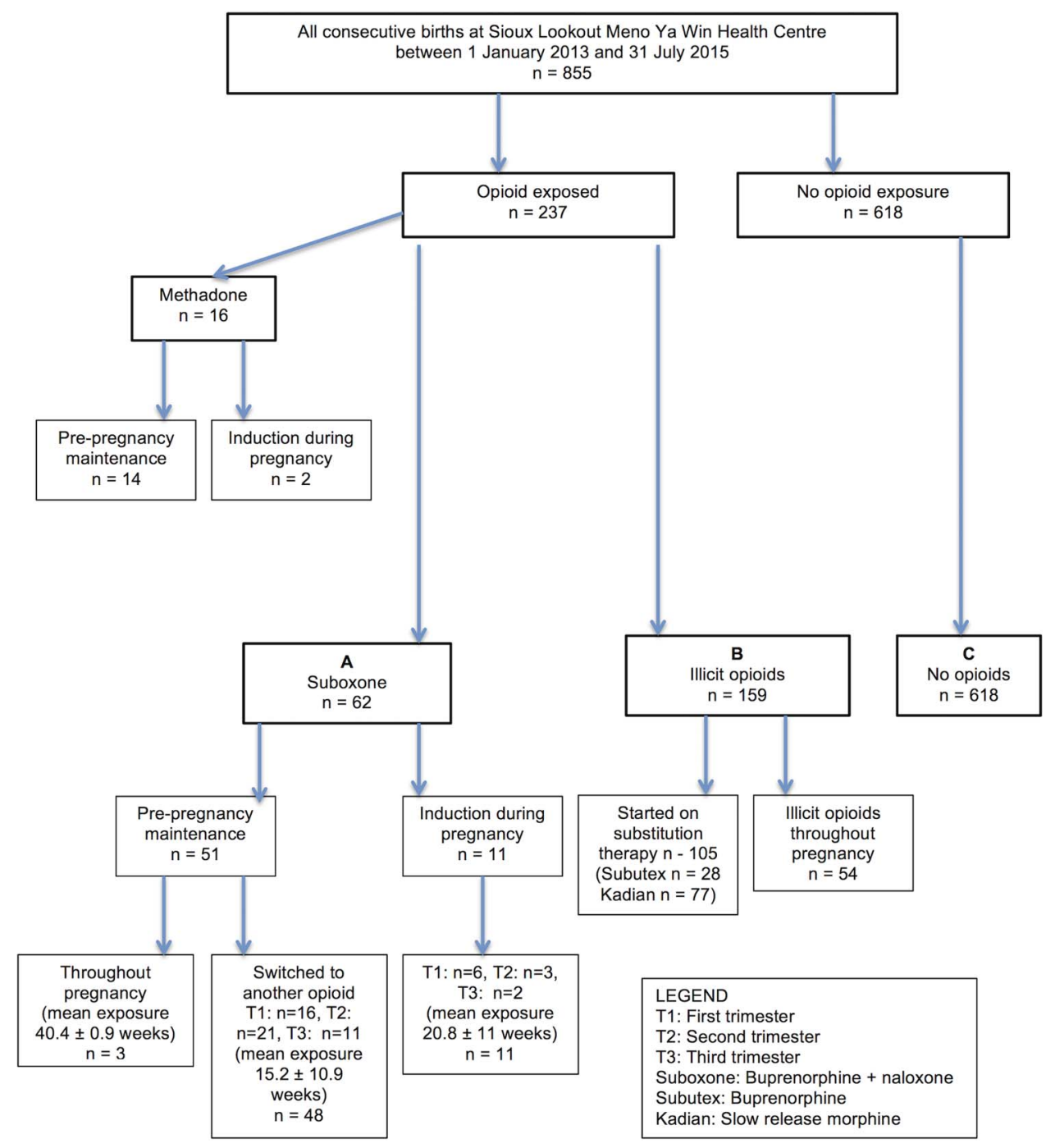

Figure 1 Patient flow chart. 
Table 1 Maternal characteristics

\begin{tabular}{|c|c|c|c|c|c|}
\hline Variable & $\begin{array}{l}\text { A } \\
\text { Bup/Nalox }(n=62)\end{array}$ & $\begin{array}{l}\text { B } \\
\text { No opioids }(n=618)\end{array}$ & $\begin{array}{l}\text { A-B } \\
p \text { Value }\end{array}$ & $\begin{array}{l}\text { C } \\
\text { Illicit opioids (n=159) }\end{array}$ & $\begin{array}{l}A-C \\
p \text { Value }\end{array}$ \\
\hline Age (years) & $25.9 \pm 4.4$ & $25.2 \pm 6.3$ & 0.21 & $25.5 \pm 4.6$ & 0.63 \\
\hline Gravida & $4.1 \pm 2.4$ & $3.3 \pm 2.3$ & 0.009 & $3.9 \pm 2.1$ & 0.54 \\
\hline Parity & $2.4 \pm 1.8$ & $1.8 \pm 2.0$ & 0.03 & $2.2 \pm 1.7$ & 0.50 \\
\hline \multicolumn{6}{|l|}{ Comorbidities } \\
\hline Type II diabetes & $5(8.1 \%)$ & $26(4.2 \%)$ & $0.19 \dagger$ & $3(1.9 \%)$ & $0.04 \dagger$ \\
\hline Gestational diabetes & $7(11.3 \%)$ & $63(10.2 \%)$ & $0.79^{*}$ & $14(8.8 \%)$ & $0.57^{*}$ \\
\hline Hypertension & $6(9.7 \%)$ & $39(6.3 \%)$ & $0.29 \dagger$ & $11(6.9 \%)$ & $0.58 \dagger$ \\
\hline HIV & 0 & 0 & & 0 & \\
\hline Hepatitis B & 0 & 0 & & $1(0.6 \%)$ & $>0.99 \dagger$ \\
\hline Hepatitis C & $3(4.8 \%)$ & $2(0.3 \%)$ & $0.006 \dagger$ & $12(7.5 \%)$ & 0.566 \\
\hline \multicolumn{6}{|c|}{ Prenatal smoking and alcohol exposure } \\
\hline \multicolumn{6}{|c|}{ Smoking, cig/day } \\
\hline None & $7(11.3 \%)$ & 327 (52.9\%) & $<0.001^{*}$ & $20(12.6 \%)$ & $0.79^{\star}$ \\
\hline $1-5$ & $33(53.2 \%)$ & 208 (33.7\%) & $0.002^{*}$ & $61(38.4 \%)$ & $0.045^{\star}$ \\
\hline $6-10$ & $15(24.2 \%)$ & $51(8.3 \%)$ & $<0.001^{*}$ & $34(21.4 \%)$ & $0.65^{\star}$ \\
\hline$>10$ & $7(11.3 \%)$ & $32(5.2 \%)$ & $0.08 \dagger$ & $44(27.7 \%)$ & $0.009 \dagger$ \\
\hline Alcohol & $11(17.7 \%)$ & $134(21.7 \%)$ & $0.47^{\star}$ & $41(25.8 \%)$ & $0.21^{*}$ \\
\hline
\end{tabular}

Data are $\mathrm{n}(\%)$ or mean \pm SD unless otherwise specified.

${ }^{*} \chi^{2}$ test was used.

†When assumptions for the $\chi^{2}$ test were not met, the Fisher's Exact test was used.

taking buprenorphine+naloxone were more likely to smoke 1-5 cigarettes per day compared to women in the control groups while women continuing to use illicit opioids were more likely to be heavy smokers $(>10$ cigarettes per day). There was no difference in alcohol consumption between the three groups.

Prenatal patterns of substance use revealed a high number of women with polysubstance use defined as the use of at least one other illicit substance among women taking buprenorphine+naloxone $(12.9 \%)$ and those continuing to use illicit opioids (53.5\%) (table 2). Treatment with buprenorphine+naloxone decreased the odds of polysubstance use in pregnancy to 0.13 (0.06 to 0.29) compared to women with ongoing illicit opioid use. The most frequent non-opioid drug of abuse was marijuana followed by cocaine (see table 2). Among women continuing to use illicit opioids during pregnancy, the majority $(59.7 \%)$ used morphine followed by oxycodone derivatives $(30.8 \%)$. The route of administration for women continuing to use illicit opioids during pregnancy was predominantly intravenous $(67.9 \%)$ and intranasal $(30.8 \%)$. Data on the primary opioid of abuse and route of administration were largely missing for women taking buprenorphine+naloxone and as a result a valid comparison cannot be made.

The primary outcome of this study is the safety of in utero exposure to buprenorphine+naloxone (table 3). For the 62 women exposed to buprenorphine+naloxone during pregnancy, the duration of exposure $($ mean $\pm \mathrm{SD})$ was $121.4 \pm 75.5$ days with a daily dose of $8.2 \pm 5.8 \mathrm{mg}$. Among parameters used to define safety of the drug, there was no difference in the birth weight, number of preterm deliveries, number of congenital malformations or number of stillbirths in women taking buprenorphine +naloxone compared to women taking no opioids during pregnancy. Women who continued to use illicit opioids had a statistically significant reduction in birth weight of $262.7 \mathrm{~g}$ compared to women treated with buprenorphine+naloxone. There were no stillbirths among the cohort taking buprenorphine+naloxone, five among the women with no opioid exposure and one among the women taking illicit opioids.

There were a total of five congenital malformations in the control group with no opioid exposure and none in the group with illicit opioid exposure. Two infants exposed to buprenorphine+naloxone had congenital malformations: one case of bilateral cleft palate and another case of atrial septal defect. The infant with the bilateral cleft palate had exposure to buprenorphine +naloxone from conception until the third trimester at which point the mother was switched to buprenorphine mono-product. The daily dose ranged from 2 to $4 \mathrm{mg}$, and the cumulative dose was $525 \mathrm{mg}$. There was alcohol exposure and smoking ( $>10$ cigarettes per day) during the pregnancy but no other illicit opioids. The infant with the atrial septal defect had exposure to buprenorphine+naloxone from the first trimester until delivery. The daily dose ranged from 2 to $4 \mathrm{mg}$, and the cumulative dose was $564 \mathrm{mg}$. There was no other smoking, drug or alcohol exposure during this pregnancy, and there is no family history of congenital heart disease. Data on prepregnancy folic acid supplementation and prepregnancy body mass index were not available for either woman. Both women were diagnosed with gestational diabetes.

Secondary neonatal outcomes (table 4) revealed no difference between cases and controls with respect to 
Table 2 Prenatal exposure to drugs of abuse

\begin{tabular}{|c|c|c|c|c|c|}
\hline & $\begin{array}{l}\text { A } \\
\text { Bup/Nalox } \\
(n=62)\end{array}$ & $\begin{array}{l}\text { B } \\
\text { No opioids } \\
(n=618)\end{array}$ & $\begin{array}{l}\text { A-B } \\
\text { p Value }\end{array}$ & $\begin{array}{l}\text { C } \\
\text { Illicit opioids } \\
(n=159)\end{array}$ & $\begin{array}{l}\text { A-C } \\
\text { p Value }\end{array}$ \\
\hline \multicolumn{6}{|l|}{ Primary opioid of abuse, $n$ (\%) } \\
\hline Morphine & $14(22.6 \%)$ & 0 & & $95(59.7 \%)$ & $<0.001^{*}$ \\
\hline Oxycodone, Percocet and OxyNeo & $5(8.1 \%)$ & 0 & & $50(31.4 \%)$ & $<0.001 \dagger$ \\
\hline Hydromorphone & $4(6.5 \%)$ & 0 & & $7(4.4 \%)$ & $0.507 \dagger$ \\
\hline Tylenol+Codeine & 0 & 0 & & $4(2.5 \%)$ & $0.578 \dagger$ \\
\hline Methadone & 0 & 0 & & $1(0.6 \%)$ & $>0.999 \dagger$ \\
\hline Suboxone & $1(1.6 \%)$ & 0 & & $9(5.7 \%)$ & $0.289 \dagger$ \\
\hline Unknown & $39(62.9 \%)$ & 0 & & $7(4.4 \%)$ & $<0.001^{*}$ \\
\hline \multicolumn{6}{|l|}{ Route of opioid administration, n (\%) } \\
\hline Intravenous & $16(25.8 \%)$ & 0 & & $108(67.9 \%)$ & $<0.001^{*}$ \\
\hline Snort/intranasal & $5(8.1 \%)$ & 0 & & $49(30.8 \%)$ & $<0.001 \dagger$ \\
\hline By mouth/per os & 0 & 0 & & $9(5.7 \%)$ & $0.064 \dagger$ \\
\hline Smoke/inhale & $1(1.6 \%)$ & 0 & & $7(4.4 \%)$ & $0.447 \dagger$ \\
\hline Not available & $42(67.7 \%)$ & 0 & & $8(5.0 \%)$ & $<0.001^{*}$ \\
\hline \multicolumn{6}{|l|}{ Frequency of opioid use, $n(\%)$} \\
\hline Occasional (two times per week or less) & $1(1.6 \%)$ & 0 & & $25(15.7 \%)$ & $0.003 \dagger$ \\
\hline Regular (three times per week or more) & $5(8.1 \%)$ & 0 & & $26(16.4 \%)$ & $0.111 \dagger$ \\
\hline Daily & $11(17.7 \%)$ & 0 & & $97(61.0 \%)$ & $<0.001^{*}$ \\
\hline Unknown & $45(72.6 \%)$ & 0 & & $11(6.9 \%)$ & $<0.001^{*}$ \\
\hline \multicolumn{6}{|l|}{ Other drugs of abuse, $\mathrm{n}(\%)$} \\
\hline Polysubstance use & $8(12.9 \%)$ & 0 & $<0.001 \dagger$ & $85(53.5 \%)$ & $<0.001^{*}$ \\
\hline Marijuana, reported & $8(12.9 \%)$ & $60(9.7 \%)$ & $0.424^{*}$ & $58(36.5 \%)$ & $0.001^{*}$ \\
\hline Marijuana, positive urine screen & $6(9.7 \%)$ & $36(5.8 \%)$ & $0.261 \dagger$ & $44(27.7)$ & $0.004 \dagger$ \\
\hline Benzodiazepines & 0 & 0 & & $3(1.9 \%)$ & $0.561 \dagger$ \\
\hline Cocaine & $1(1.6 \%)$ & 0 & $0.091 \dagger$ & $10(6.3 \%)$ & $0.299 \dagger$ \\
\hline Ecstasy & 0 & 0 & & $1(0.6 \%)$ & $>0.999 \dagger$ \\
\hline
\end{tabular}

Table 3 Primary outcomes

\begin{tabular}{|c|c|c|c|c|c|}
\hline & $\begin{array}{l}\text { A } \\
\text { Bup/Nalox }(n=62)\end{array}$ & $\begin{array}{l}\text { B } \\
\text { No opioids }(n=618)\end{array}$ & $\begin{array}{l}\text { A-B } \\
\text { p Value }\end{array}$ & $\begin{array}{l}\text { C } \\
\text { Illicit opioids }(n=159)\end{array}$ & $\begin{array}{l}\text { A-C } \\
\text { p Value }\end{array}$ \\
\hline \multicolumn{6}{|l|}{ Primary outcomes } \\
\hline Preterm delivery, $\mathrm{n}(\%) \ddagger$ & $2(3.2 \%)$ & $26(4.21 \%)$ & $>0.999 \dagger$ & $7(4.4 \%)$ & $>0.999 \dagger$ \\
\hline Birth weight $(\mathrm{g})$, mean \pm SD $\ddagger$ & $3541 \pm 540$ & $3553 \pm 569$ & $0.924^{*}$ & $3274 \pm 551$ & $<0.001^{*}$ \\
\hline Congenital anomalies, n (\%) & $2(3.2 \%)$ & $5(0.8 \%)$ & $0.127 \dagger$ & 0 & $0.078 \dagger$ \\
\hline Stillbirths & 0 & $5(0.8 \%)$ & $>0.999 \dagger$ & $1(0.6 \%)$ & $>0.999 \dagger$ \\
\hline
\end{tabular}

gestational age at the time of delivery, Apgar scores, NAS Scores and NAS treatment. Secondary maternal outcomes (table 4) showed that mothers exposed to buprenorphine+naloxone stayed in hospital an extra 1.1 days compared to mothers with no opioid exposure in pregnancy. There was no statistically significant difference in length of stay between cases and illicit opioid using controls. There was no difference in the number of caesarean sections, postpartum haemorrhages, out of hospital deliveries or transfers to tertiary care hospitals for cases compared to controls.

\section{DISCUSSION}

Almost one-third of the study population were exposed to opioids during pregnancy, but only $5.6 \%$ were on opioid agonist treatment prior to pregnancy. There was no evidence of teratogenicity or adverse pregnancy outcomes in a cohort of 62 women exposed to buprenorphine+naloxone during pregnancy compared to women who had no opioid exposure during pregnancy. Two cases of congenital malformations were identified in women exposed to buprenorphine+naloxone. One case of bilateral cleft lip and palate included a significant 
Table 4 Secondary outcomes

\begin{tabular}{|c|c|c|c|c|c|}
\hline & $\begin{array}{l}\text { A } \\
\text { Bup/Nalox }(n=62)\end{array}$ & $\begin{array}{l}\text { B } \\
\text { No opioids }(n=618)\end{array}$ & $\begin{array}{l}\text { A-B } \\
\text { p Value }\end{array}$ & $\begin{array}{l}\text { C } \\
\text { Illicit opioids ( } n=159)\end{array}$ & $\begin{array}{l}\text { A-C } \\
\text { p Value }\end{array}$ \\
\hline \multicolumn{6}{|l|}{ Neonatal outcomes } \\
\hline Gestational age at birth, mean $\pm S D$ & $38.7 \pm 1.5$ & $38.9 \pm 1.5$ & 0.405 & $38.6 \pm 1.5$ & 0.686 \\
\hline Apgar $1 \mathrm{~min}, \operatorname{mean} \pm \mathrm{SD}$ & $8.7 \pm 0.8$ & $8.6 \pm 1.3$ & 0.780 & $8.6 \pm 1.2$ & 0.407 \\
\hline Apgar 5 min, mean $\pm S D$ & $9.0 \pm 0.4$ & $8.9 \pm 0.8$ & 0.761 & $8.9 \pm 0.8$ & 0.421 \\
\hline \# NAS Score $>7$ & $1(1.6 \%)$ & 0 & & $11(6.9 \%)$ & $0.186 \dagger$ \\
\hline Treated for NAS & $1(1.6 \%)$ & 0 & & $9(5.7 \%)$ & $0.289 \dagger$ \\
\hline Males & $38(61.3 \%)$ & 325 (52.6\%) & $0.229^{*}$ & $81(50.9 \%)$ & $0.166^{*}$ \\
\hline \multicolumn{6}{|l|}{ Maternal outcomes } \\
\hline Caesarean section & $14(22.6 \%)$ & $151(24.4 \%)$ & $0.746^{*}$ & $42(26.4 \%)$ & $0.556 \dagger$ \\
\hline Postpartum haemorrhage & $10(16.1 \%)$ & $61(9.9 \%)$ & $0.124^{\star}$ & $14(8.8 \%)$ & $0.116 \dagger$ \\
\hline Length of maternal stay in hospital & $3.1 \pm 1.5$ & $2.0 \pm 1.1$ & $<0.001$ & $2.8 \pm 1.9$ & 0.235 \\
\hline Out of hospital deliveries & $1(1.6 \%)$ & $11(1.8 \%)$ & $>0.999 \dagger$ & $3(1.9 \%)$ & $>0.999 \dagger$ \\
\hline Transfer to tertiary care centre & $2(3.2 \%)$ & $13(2.1 \%)$ & $0.639 \dagger$ & $5(3.1 \%)$ & $>0.999 \dagger$ \\
\hline
\end{tabular}

confounder in the form of in utero alcohol exposure. The second was a case of an atrial septal defect-one of the most common congenital malformations occurring in 1 in 1500 live births. ${ }^{17}$ Data regarding other confounders such as folic acid supplementation, exposure to other medications and obesity were not available. In spite of these limitations, the rate of congenital malformations was not significantly different compared to the control group.

Women who continued to use illicit opioids during pregnancy did have babies with a statistically significant lower birth weight compared to those women taking buprenorphine+naloxone. The rate of congenital malformations was not above the rate in the general population although the cohort exposed to buprenorphine +naloxone is small and therefore may give a biased estimation of this risk. Exposure to buprenorphine+naloxone also had no observable impact on Apgar scores or treatment for NAS. The low rates of NAS observed in this study may be accounted for by several factors. First, the majority of community-based opioid agonist programmes included in the study use a low dose treatment protocol where the average maintenance dose of buprenorphine+naloxone is less than $8 \mathrm{mg}$ daily. Second, for those women who continue to use illicit opioids, nondaily, binge use is the predominant pattern of opioid use and short acting opioids (morphine and oxycodone) are the most common opioids of abuse. ${ }^{17}$ Furthermore, opioid tapering during pregnancy, with long-acting morphine, is a common practice in this setting and results in lower doses of opioids at the time of delivery. ${ }^{18}$ Finally, all infants room-in with their mother after delivery and breastfeeding and supportive care are encouraged, all of which are beneficial in the management of NAS.

This study represents the largest cohort of women exposed to buprenorphine+naloxone in pregnancy and contains detailed information about the daily dose, cumulative dose and exposure time with respect to each trimester of pregnancy. We present an assessment of key safety parameters for mother and infant. A harm reduction strategy is applied in our catchment area as it has been shown that there are better pregnancy outcomes when a woman is part of a treatment programme compared to ongoing use of illicit opioids. Many of the women in this study were inducted on to buprenorphine+naloxone during pregnancy rather than the buprenorphine mono-product. In our jurisdiction, the buprenorphine mono-product is available through a special access programme with lengthy delays between applying for an exemption and receipt of the drug, whereas buprenorphine+naloxone is readily available in communities as part of community-based harm reduction programmes. As a result, women are induced onto buprenorphine+naloxone and maintained on this drug until such time that the buprenorphine mono-product becomes available.

Two small retrospective cohort studies are reported in the literature that look at the effect of buprenorphine +naloxone on pregnancy outcomes. ${ }^{19-21}$ The first compared 10 women who took buprenorphine+naloxone in pregnancy to women who took buprenorphine or methadone in six other studies with similar outcome measures. No differences in maternal outcomes or neonatal outcomes were observed with the exception of head circumference which was larger among infants exposed to buprenorphine+naloxone compared to infants who were exposed to methadone withdrawal in utero. ${ }^{19} 20$ The second retrospective cohort study compared 31 women who took buprenorphine+naloxone in pregnancy to a control group of 31 women who took methadone in pregnancy. The study excluded stillbirth and congenital malformations as well as women who received treatment for $<30$ days prior to delivery. Again, no significant differences in maternal or neonatal outcomes were observed. Infants exposed to methadone were born at an earlier gestational age than buprenorphine+naloxone, but the average gestational age for both was $>38$ weeks. ${ }^{21}$ 
Buprenorphine+naloxone is an efficacious treatment for opioid dependence with the advantage of a lower overdose risk and ease of prescribing when compared to methadone. ${ }^{22}$ Owing to these factors, it is being used widely in opioid treatment programmes in rural and remote settings where methadone treatment is not available. ${ }^{23}$ Currently women participating in buprenorphine+naloxone treatment programmes who become pregnant are advised to switch to the buprenorphine mono-product out of theoretical concerns of the risk of naloxone to the developing fetus. This study demonstrates no increase rate of congenital malformations, stillbirth or low birth weight for women exposed to buprenorphine+naloxone. Anecdotally women who are stable on buprenorphine+naloxone prior to pregnancy express concern about changing medications and often reluctantly switch to the buprenorphine monoproduct on the recommendation of their care provider.

Results from this study support the safety of buprenorphine+naloxone in pregnancy and provide evidence for inclusion of pregnancy as an indication for buprenorphine+naloxone therapy. A policy change of this nature would increase access to care for many women residing in rural and remote settings or where methadone is not an option. Ongoing research and postmarket surveillance would be required to assess rare outcomes. Furthermore, longitudinal studies of the infants exposed to buprenorphine in utero should be performed to assess developmental outcomes. This would add valuable information on the safety of this medication in pregnancy.

\author{
Author affiliations \\ ${ }^{1}$ Northern Ontario School of Medicine, Thunder Bay, Ontario, Canada \\ ${ }^{2}$ School of Public Health, University of Saskatchewan, Saskatoon, \\ Saskatchewan, Canada \\ ${ }^{3}$ Anishinaabe Bimaadiziwin Research Program, Sioux Lookout, Ontario, \\ Canada \\ ${ }^{4}$ Integrated Pregnancy Program, Sioux Lookout Meno Ya Win Health Centre, \\ Sioux Lookout, Canada
}

Contributors NAJ and CE contributed to data analysis and manuscript preparation. JB-B and $\mathrm{KL}$ involved in data collection and provided approval of final draft. JD and LGF contributed to data collection and manuscript preparation. LK involved in data collection, data analysis and manuscript preparation.

Funding This research received no specific grant from any funding agency in the public, commercial or not-for-profit sectors.

Competing interests None declared.

Ethics approval Sioux Lookout Meno Ya Win Health Centre.

Provenance and peer review Not commissioned; externally peer reviewed.

Data sharing statement Source data will not be shared publicly as this was not stipulated in the original ethics approval. Data may be obtained by emailing Ikelly@mcmaster.ca.

Open Access This is an Open Access article distributed in accordance with the Creative Commons Attribution Non Commercial (CC BY-NC 4.0) license, which permits others to distribute, remix, adapt, build upon this work noncommercially, and license their derivative works on different terms, provided the original work is properly cited and the use is non-commercial. See: http:// creativecommons.org/licenses/by-nc/4.0/

\section{REFERENCES}

1. Dooley R, Dooley J, Antone I, et al. Narcotic tapering in pregnancy using long-acting morphine: an 18 month year prospective study in Northwest Ontario. Can Fam Physician 2015;61:e88-95.

2. Walker R, Cromarty H, Kelly L, et al. Achieving Cultural Safety in Aboriginal Health Services: implementation of a cross-cultural safety model in a hospital setting. Divers Health Care 2009;6:11-22.

3. Jones HE, Martin PR, Heil SH, et al. Treatment of opioid-dependent pregnant women: clinical and research issues. J Subst Abuse Treat 2008;35:245-59.

4. Winklbaur B, Kopf N, Ebner N, et al. Treating pregnant women dependent on opioids is not the same as treating pregnancy and opioid dependence: a knowledge synthesis for better treatment for women and neonates. Addiction 2008;103:1429-40.

5. Katt M, Chase C, Samokhvalov A, et al. Feasibility and outcomes of a community-based taper-to-low-dose-maintenance suboxone treatment program for prescription opioid dependence in a remote First Nations community in Northern Ontario. $J$ Aborig Health 2012;9:52-9.

6. World Health Organization. Guidelines for the identification and management of substance use and substance use disorders in pregnancy. Geneva, Switzerland: World Health Organization, 2014:14.

7. Wong S, Ordean A, Kahan M. Substance abuse in pregnancy. J Obstet Gynaecol Can 2011;33:367-84.

8. American College of Obstetricians and Gynecologists. Opioid abuse, dependence and addiction in pregnancy. Committee opinion 524 Obstet Gynecol 2012;119:1070-1.

9. Jones HE, Kaltenbach K, Heil SH, et al. Neonatal abstinence syndrome after methadone or buprenorphine exposure. $N$ Engl J Med 2010;363:2320-31.

10. Daily Med. Suboxone tablet (product monograph). Bethesda (MD): National Institutes of Health, 2013. http://dailymed.nlm.nih.gov/ dailymed/lookup.cfm?setid=4b9b43c4-293e-4323-a1a19a2f6a16ac39 (accessed 23 Aug 2013).

11. Daily Med. Suboxone soluble film (product monograph). Bethesda (MD): National Institutes of Health, 2013. http://dailymed.nIm.nih.gov/ dailymed/lookup.cfm? setid=8a5edcf9-828c-4f97-b671-268ab13a8ecd (accessed 23 Aug 2013).

12. Rementeriá JL, Nunag NN. Narcotic withdrawal in pregnancy: stillbirth incidence with a case report. Am J Obstet Gynecol 1973;116:1152-6.

13. Stern R. The pregnant addict: a study of 66 case histories, 19501959. Am J Obstet Gynecol 1966;94:253-7.

14. Daily Med. Naloxone hydrochloride (product monograph). Bethesda (MD): National Institutes of Health, 2013. http://dailymed.nlm.nih.gov/ dailymed/lookup.cfm?setid=d524c0e5-a7c2-40b2-9eed-2caf71c787dc (accessed 23 Aug 2013).

15. Food and Drug Administration. Reviewer guidance evaluating the risks of drug exposure in human pregnancies. Rockville (MD): Food and Drug Administration, 2005:4.

16. Indigenous and Northern Affairs Canada. Aboriginal women in Canada: a statistical profile from the 2006 census. Ottawa (ON): Government of Canada, 2012. https://www.aadnc-aandc.gc.ca/eng/ 1331664678840/1331838092221

17. Porter CJ, Feldt RH, Edwards WD, et al. Chapter 27: atrial septal defects. In: Allen HD, Gutgesell HP, Clark EB et al. eds. Moss \& Adams' heart disease in infants, children \& adolescents: including the fetus and young adults. 8th edn. Philadelphia (PA): Lippincott, Williams and Wilkins, 2013 (accessed 29 May 2016).

18. Kelly L, Guilfoyle J, Dooley J, et al. incidence of narcotic abuse during pregnancy in Northwestern Ontario: three-year prospective cohort study. Can Fam Physician 2014;60:e493-8.

19. Debelak K, Morrone WR, O'Grady KE, et al. Buprenorphine +naloxone in the treatment of opioid dependence during pregnancyinitial patient care and outcome data. Am J Addict 2013;22:252-4.

20. Lund IO, Fisher G, Welle-Strand G, et al. A Comparison of buprenorphine+naloxone to buprenorphine and methadone in the treatment of opioid dependence during pregnancy: maternal and neonatal outcomes. Subst Abuse 2013;7:61-74.

21. Wiegand SL, Stringer EM, Stuebe AM, et al. Buprenorphine and naloxone compared with methadone treatment in pregnancy. Obstet Gynecol 2015;125:363-8.

22. Mattick RP, Breen C, Kimber J, et al. Buprenorphine maintenance versus placebo or methadone maintenance for opioid dependence. Cochrane Database Syst Rev 2014;(2):CD002207.

23. Jumah NA, Graves L, Kahan M. The management of opioid dependence in pregnancy in rural and remote settings. CMAJ 2015;187:E41-6. 